\title{
Slow Aging Dynamics and Avalanches in a Gold-Cadmium Alloy Investigated by X-Ray Photon Correlation Spectroscopy
}

\author{
L. Müller, ${ }^{1 *}$ M. Waldorf, ${ }^{1}$ C. Gutt, ${ }^{2}$ G. Grübel,${ }^{2}$ A. Madsen,,${ }^{3, \dagger}$ T. R. Finlayson, ${ }^{4}$ and U. Klemradt ${ }^{1, \hbar}$ \\ ${ }^{1}$ II. Physikalisches Institut, RWTH Aachen University, D-52056 Aachen, Germany \\ ${ }^{2}$ Deutsches Elektronen-Synchrotron DESY, Notkestraße 85, D-22607 Hamburg, Germany \\ ${ }^{3}$ European Synchrotron Radiation Facility, Bôite Postale 220, F-38043 Grenoble, France \\ ${ }^{4}$ School of Physics, University of Melbourne, Victoria 3010, Australia
}

(Received 30 September 2010; revised manuscript received 4 July 2011; published 1 September 2011)

Results of a X-ray photon correlation spectroscopy experiment on the very weakly first order martensitic transformation of $\mathrm{a} \mathrm{Au}_{50.5} \mathrm{Cd}_{49.5}$ single crystal are presented. Slow non-equilibrium-dynamics are observed in a narrow temperature interval in the direct vicinity of the otherwise athermal phase transformation. These dynamics are associated with the martensite-aging effect. The dynamical aging is accompanied by an avalanchelike behavior which is identified with an incubation-time phenomenon.

PACS numbers: 64.70.kd, 41.60.Ap, 66.30.- h, 81.30.Kf

A martensitic transformation (MT) is a structural phase transformation and belongs to the class of ferroic phase transformations [1,2]. MTs are of great economical interest as they play a key role in steel hardening and if of weakly first order character, they constitute the physical basis for shape memory alloys, intelligent materials that can be used as functional materials with many different applications, see, e.g., [3]. Martensitic steel hardening has been used for over a thousand years and the microstructure of martensitic steel has been described for the first time over a century ago [4]. In the following decades a rich field of research about all kinds of martensitic alloys has developed, however key features of the transformation process remain unresolved $[5,6]$. Of particular interest is the elusive nucleation process and the detailed mechanism of the MT, namely, the intermediate structures during the transformation itself $[7,8]$.

MTs are discontinuous phase transformations and in most cases they are of athermal character, hence no explicit time dependence is expected. Therefore, it came as a surprise when a sudden occurrence or progression of the MT at fixed temperatures $T>M_{\mathrm{S}}\left(M_{\mathrm{S}}\right.$ denotes the transformation start temperature on cooling at which a spontaneous distortion occurs) and a subsequent two-phase coexistence region was observed [9]. In the following decades several observations of this so called incubation time have been made [10].

Another puzzling and controversial phenomenon is the so called martensite aging. It refers to a rise of the reversetransformation temperature (martensite stabilization) in conjunction with the buildup of a restoring force that reverts the martensite to its original form after a pseudoplastic deformation (rubberlike behavior) [11]. Depending on the material system the time needed to make the effect noticeable ranges from seconds in In-Tl alloys to months in $\mathrm{Cu}-\mathrm{Al}-\mathrm{Ni}$ alloys [12]. A ratio close to 1 between $M_{\mathrm{s}}$ and the melting temperature gives rise to fast aging, and alloys with a lower ratio show slow martensite aging. As a result diffusion was assumed to play a key role and finally a model consistently explaining martensite stabilization as well as the rubberlike behavior was proposed $[11,13]$. In short, this so called symmetry-conforming short-rangeordering (SC SRO) model explains martensite aging with a diffusive rearrangement of lattice defects according to the (reduced) symmetry in the new phase (Fig. 1). Even though initial data supported the SC SRO model [14], direct observations of the underlying diffusive dynamics have been missing up to now.

The material system $\mathrm{Au}_{50.5} \mathrm{Cd}_{49.5}$ was chosen to observe the diffusive dynamics, as proposed in the SC SRO model, in conjunction with the test of a previously suggested critical slowing-down scenario at temperatures above the MT [10]. Close to the stoichiometric composition this alloy exhibits a MT and the associated shape memory effect. The latent heat of the MT is only $(2.09 \pm 0.2) \mathrm{J} / \mathrm{g}$, hence the

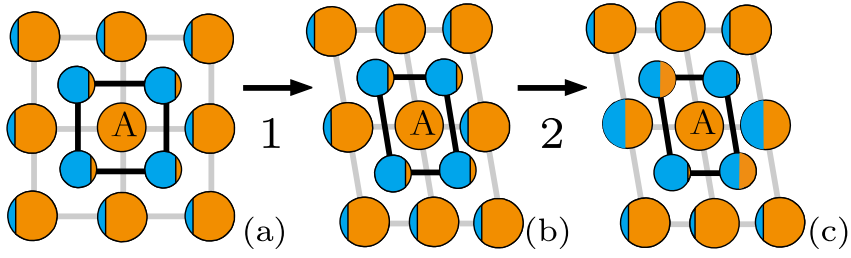

FIG. 1 (color online). Schematic representation of the martensitic transformation of an $\mathrm{AB}$ alloy with subsequent martensite aging according to the SC SRO model. The transformation (1) from the high symmetry (a) to the martensite phase (b) is diffusionless and happens on very fast time scales. If the sample is held at a fixed temperature the transformation may occur or proceed after a certain incubation time. Subsequently, the diffusive aging process (2) rearranges the average defect arrangement until it corresponds to the reduced symmetry (c). The two colors represent atoms $\mathrm{A}$ and $\mathrm{B}$, respectively, and their proportions indicate the probability of finding an atom of type A or B at the respective position. See $[11,13]$ for further details. 
MT of this alloy should be close to a second order transformation [15] and critical slowing down is expected to be quite pronounced. At $M_{\mathrm{s}}$ the symmetry changes from a cubic B2 $(a=3.33 \AA)$ to a trigonal $\zeta_{2}^{\prime}$ structure [16]. The system shows strong precursor effects of the MT [17-19] supporting a possible connection to a second order transformation and the aging effect is developing within a time of about half an hour [12].

$\mathrm{X}$-ray photon correlation spectroscopy (XPCS) is used to observe dynamics at atomic length scales. The coherent $\mathrm{x}$ rays used by this technique provide a sensitive probe of structural changes within the sample through the observation of a time-dependent speckle pattern. XPCS has previously been applied to the study of dynamics in various systems including dynamics of phase transformations in soft and hard condensed matter [20-24].

The experiment was carried out at beam line ID 10A at the ESRF in Grenoble employing a standard Bragg scattering geometry [Fig. 2(a)] to observe the fluctuations in
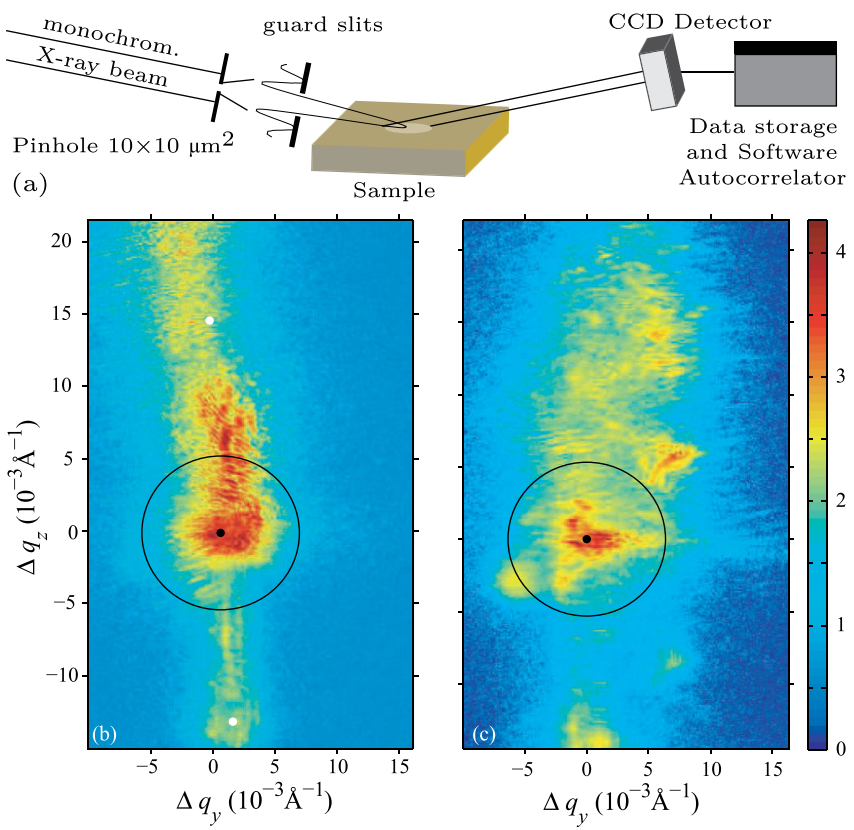

FIG. 2 (color online). (a) Schematic setup for x-ray photon correlation spectroscopy measurements in Bragg geometry. See text for details. (b) Diffraction image of the (lllll) Bragg reflection summed over 30 exposures at temperatures of $330 \mathrm{~K}$ and $305.3 \mathrm{~K}=M_{\mathrm{s}}-0.05 \mathrm{~K}$ (c). The white markings in the high temperature image show the regions where the low temperature Bragg reflections will be located. The intensities of these regions vary gradually with temperature until the diffraction image changes drastically at the transformation temperature. In both pictures the circle indicates the region of interest for which the correlation functions are calculated and the black dots indicates the center of the Bragg reflection defined by the highest intensity pixels. The $q$ values have been calculated with respect to this reference. The $q_{z}$ and the logarithmic color scales apply to both images. the bulk of the Au-Cd single crystal. The $\left(\begin{array}{lll}0 & 0 & 1\end{array}\right)$ surface was polished and the symmetric $\left(\begin{array}{lll}0 & 0 & 1\end{array}\right)$ Bragg reflection $\left(\theta_{B}=13.4^{\circ}\right)$ chosen for the experiment. To achieve sufficient transversal coherence the monochromatic x-ray beam was collimated by a small pinhole aperture $\left(10 \times 10 \mu \mathrm{m}^{2}\right)$ placed approximately $50 \mathrm{~cm}$ upstream of the sample. Additional guard slit apertures were employed to suppress stray scattering from the pinhole. The radiation diffracted by the sample is detected by a CCD camera (Princeton Instruments, directly illuminated chip, $1242 \times 1152$ pixels, pixel size $22.5 \times 22.5 \mu \mathrm{m}^{2}$ ) placed $135 \mathrm{~cm}$ downstream of the sample. An area of $401 \times 650$ pixels, containing both high- and low-temperature-phase Bragg reflections, was read out from the camera. The correlation function is then calculated by a software autocorrelator. To avoid an elevated fluorescence background the x-ray energy was set to $8 \mathrm{keV}$, which in addition provides high coherent photon flux. The time characteristics of the $\left(\begin{array}{lll}0 & 0 & 1\end{array}\right)$ Bragg reflection which is sensitive to the structural transformation were recorded for temperatures in the vicinity of the MT. Data were taken with a time resolution of $1.4 \mathrm{~s}$ ( $0.2 \mathrm{~s}$ exposure time followed by $1.2 \mathrm{~s}$ readout time). For each temperature a data set typically contains 1100 exposures. During the experiment the transformation temperature was determined by a dramatic change of the speckle pattern to be $M_{\mathrm{s}}=305.35 \mathrm{~K}$. A nominal temperature stability of $\pm 3 \mathrm{mK}$ in the relevant temperature range from $260 \mathrm{~K}$ to $370 \mathrm{~K}$ was provided by a dedicated sample chamber. For the measurements the temperature was lowered from approximately $5 \mathrm{~K}$ above $M_{\mathrm{s}}$ in steps of $1 \mathrm{~K}$ and in steps of $0.1 \mathrm{~K}$ for temperatures below $306 \mathrm{~K}$ until the MT occurred. We estimate that the temperature drift of the sample during data acquisition was significantly smaller than $0.03 \mathrm{~K}$ for all temperatures investigated.

Upon approaching the MT a gradual change in the recorded speckle images can be observed. This effect starts to be observable even at temperatures $30 \mathrm{~K}$ above $M_{\mathrm{s}}$, indicating structural rearrangements in the sample already well above the phase transition [Fig. 2(b) and 2(c)], in agreement with other studies on shape memory alloys $[25,26]$. This continuous precursor is reminiscent of a second order behavior. It is excluded that isolated regions transforming individually at higher temperatures owing to some structural or chemical inhomogeneities could be responsible for this behavior as only an area smaller than a single martensitic domain is illuminated. The lateral domain size has been measured with optical microscopy to be of the order of several $100 \mu \mathrm{m}$ [27] whereas the illuminated sample volume is $10 \times 45 \times 2 \mu \mathrm{m}^{3}$. In contrast to the extended precursor at the phase transition the diffraction image changes abruptly within a temperature interval of less than $0.05 \mathrm{~K}$, pointing towards a distinct first order behavior.

The data are evaluated by calculating the time averaged one-time correlation function 


$$
\begin{aligned}
g_{2}(q, \tau) & =\frac{\langle I(t) I(t+\tau)\rangle_{t}}{\langle I(t)\rangle_{0 \leq t \leq t_{\mathrm{run}}}-\tau|I(t)\rangle_{\tau \leq t \leq t_{\mathrm{run}}}} \\
& =1+A\left|f_{1}(\tau)\right|^{2},
\end{aligned}
$$

where $A$ denotes the speckle contrast and $f_{1}(\tau)$ is the intermediate scattering function.

In the case of equilibrium dynamics the resulting $f_{1}$ can be described by a Kohlrausch-Williams-Watts (KWW) exponential form

$$
f_{1}(\tau)=\exp \left[-\left(\tau / \tau_{0}\right)^{\beta}\right]
$$

with a characteristic time scale $\tau_{0}$ and the KWW exponent $\beta$.

In a second step the two-times correlation function [28]

$$
C_{2}\left(q, t_{1}, t_{2}\right)=\frac{I\left(t_{1}\right) I\left(t_{2}\right)}{\left\langle I\left(t_{1}\right)\right\rangle_{t}\left\langle I\left(t_{2}\right)\right\rangle_{t}}
$$

is calculated to characterize non-equilibrium-dynamics. For temperatures other than $305.3 \mathrm{~K}$ this is not possible due to the extremely long time scales leading to dominating noise. Particularly at the temperature of $305.3 \mathrm{~K}$, close to the MT, dynamics can be seen even by eye as a relative movement of some features of the speckle pattern (see supporting online material [29]). As the Bragg peak is a measure of the order parameter, all correlation functions were calculated in a circular region around the $\left(\begin{array}{lll}0 & 0 & 1\end{array}\right)$ reflection [Fig. 2(b)].

The time scales and corresponding KWW exponents extracted from the one-time correlation functions (Fig. 3) for $T \neq 305.3 \mathrm{~K}$ and from the two-times correlation function [Fig. 4(a)] for $T=305.3 \mathrm{~K}$ are shown in Fig. 4(b). In the latter case one-time correlation functions were calculated by binning the two-times correlation function over ten time steps $(\approx 14 \mathrm{~s})$ to reduce noise, see, e.g., [30]. The times at which these correlation functions start are chosen

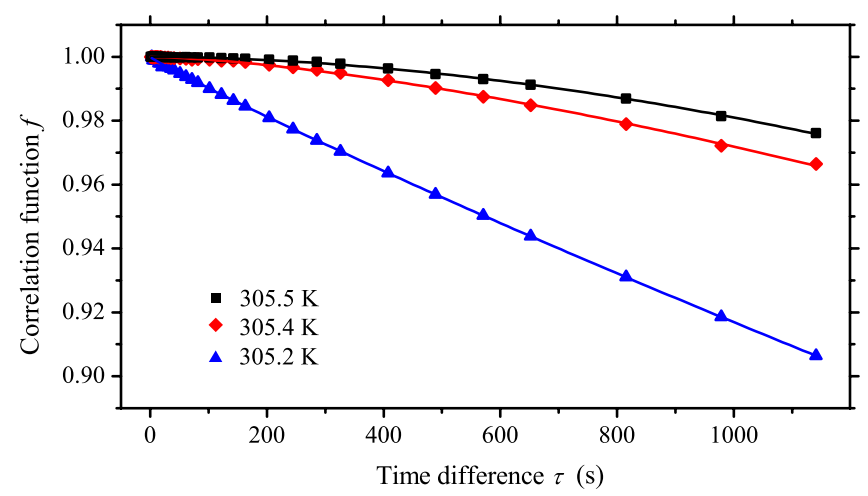

FIG. 3 (color online). One-time correlation functions at temperatures close to the martensitic transformation. At temperatures higher than $M_{s}=305.35 \mathrm{~K}$ the correlation functions show compressed behavior but at temperatures below $M_{s}$ the behavior is almost exponential. The solid lines represent fits to the data. such that they have a maximum length before crossing one of the cuts produced by avalanches and are evaluated only up to this point.

At temperatures higher than $M_{\mathrm{s}}$ the one-time correlation functions show dynamics with KWW exponents up to a value of $\beta \approx 2$. These compressed exponential forms of $f_{1}$ indicate strain dominated dynamics with long time scales in the range of $10^{4} \mathrm{~s}$. This behavior can be expected as a MT always implies the presence of strain fields due to the change in lattice symmetry.
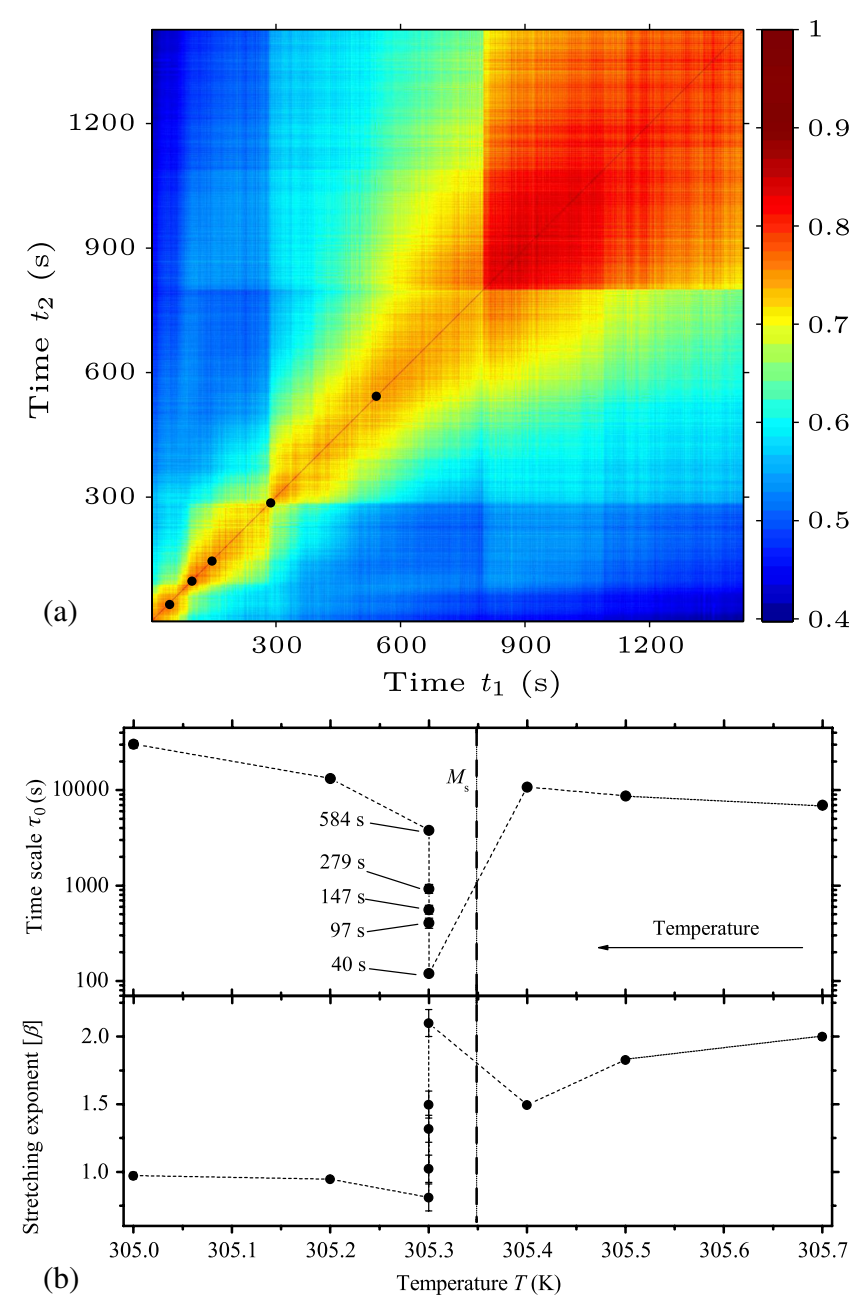

FIG. 4 (color online). (a) Two-times correlation function $C_{2}$ at a temperature of $305.3 \mathrm{~K}$ calculated via Eq. (4) for a circular region with a radius of 50 pixels around the center of the high temperature Bragg reflection. Note the divergent color contours showing non-equilibrium dynamics and the sharp-cut divisions in several rectangles due to avalanche dynamics. The latter is caused by avalanches in the sample occurring at $65,89,285$, and $798 \mathrm{~s}$. The black dots indicate the starting times of the extracted one-time correlation functions. (b) Time scales $\tau_{0}$ and corresponding KWW exponents $\beta$ extracted from one-time correlation functions or-in the case of $305.3 \mathrm{~K}$-from the two-times correlation function at specific times marked in (a). See text for details. 
At a temperature close to $M_{s}(305.3 \mathrm{~K})$ the one-time correlation function is no longer a good measure of the now heterogenous dynamics as the corresponding two-times correlation function [Fig. 4(a)] shows that the time scales of the dynamics increase continuously with waiting time and additionally displays sharp cuts which points toward an avalanchelike behavior in the sample, identified with a sudden progression of the phase transition. The associated time scale cannot be resolved in this experiment due to the frame rate limitation. At those times a major change occurs in the speckle pattern [29] leading to the observed instantaneous decorrelation. These intermittent progression of the transformation is identified with an incubation time effect. This conclusion is based on the resemblance with the dynamics specific for all incubation time effects, see [10] and references herein. This has also been measured in this sample [27] with an optical laser light reflection technique similar to [31]. Apart from this additional phenomenon the dynamics suddenly become faster by almost 2 orders of magnitude (compared to those for $T=305.4 \mathrm{~K}$ ) and the stress is gradually relaxed until the KWW exponent reaches a value of $\lesssim 1$. Simultaneously, the relaxation time increases steadily with time and attains again $\approx 10^{4} \mathrm{~s}$ after about $10 \mathrm{~min}$ waiting time. The observed dynamics are attributed to the martensite aging effect and the measurements present a direct observation of the diffusional dynamics causing this effect, as predicted in the framework of the SC SRO model.

At temperatures lower than $M_{\mathrm{s}}$ the dynamics remain diffusive $(\beta \approx 1)$ with long time scales in the $10^{4} \mathrm{~s}$ range. For temperatures below $305 \mathrm{~K}$ time constants larger than $10^{5} \mathrm{~s}$ are calculated which however can also be induced by the experimental setup itself and therefore allow no assertion about real dynamics in the sample. The observed increasing of the time scales after the transformation is in qualitative accordance with the fact that martensite aging becomes observable relatively quickly but then develops over a much longer time [32].

In conclusion, XPCS was successfully applied to measure time-dependent phenomena in an athermal martensitic shape memory alloy. At the transformation temperature we find avalanche dynamics caused by the incubation time effect alongside with slow dynamics explained by the SC SRO model of martensite aging. Furthermore, the correlated observation of both effects points towards a possible so far unknown connection of both phenomena. Additionally, the absence of any avalanche dynamics at different temperatures than at $305.3 \mathrm{~K}$ implies that the incubation-time phenomenon is limited to a rather small temperature interval around $M_{\mathrm{s}}$ although precursors of the martensitic phase are observable already at high temperatures [Fig. 2(b) and 2(c)] and signs of the high temperature phase remain visible even some $10 \mathrm{~K}$ below $M_{\mathrm{s}}$ [27]. Finally, no signature of critical slowing down was seen in the here observed $q$ range.
*Present address: Deutsches Elektronen-Synchrotron DESY, Notkestraße 85, D-22607 Hamburg, Germany.

${ }^{\dagger}$ Present address: European XFEL, Albert-Einstein-Ring 19, 22761 Hamburg, Germany.

*klemradt@physik.rwth-aachen.de

[1] K. Bhattacharya, Microstructure of Martensite (Oxford, Oxford, 2003), ISBN 978-0198509349.

[2] K. Aizu, Phys. Rev. B 2, 754 (1970).

[3] M. Kohl, Shape Memory Microactuators (Springer, New York, 2004), ISBN 978-3540206354.

[4] A. Martens, Zeitschr. Ver. dt. Ingen. 22, 205 (1878).

[5] M. Aspelmeyer, U. Klemradt, H. Abe, S. C. Moss, and J. Peisl, Mater. Sci. Eng. A 273-275, 286 (1999).

[6] T. Kakeshita, T. Saburi, and K. Shimizu, Mater. Sci. Eng. A 273-275, 21 (1999).

[7] J. Buschbeck, I. Opahle, M. Richter, U. K. Rößler, P. Klaer, M. Kallmayer, H. J. Elmers, G. Jakob, L. Schultz, and S. Fähler, Phys. Rev. Lett. 103, 216101 (2009).

[8] T. Kakeshita, K. Kuroiwa, K. Shimizu, T. Ikeda, A. Yamagishi, and M. Date, Mater. Trans., JIM 34, 415 (1993), http://www.jim.or.jp/journal/e/34/05/415.html.

[9] H. G. Smith, Phys. Rev. Lett. 58, 1228 (1987).

[10] L. Müller, U. Klemradt, and T. R. Finlayson, Mater. Sci. Eng. A 438-440, 122 (2006).

[11] X. Ren and K. Otsuka, Nature (London) 389, 579 (1997).

[12] X. Ren and K. Otsuka, Phase Transit. 69, 329 (1999).

[13] K. Otsuka and X. Ren, Scr. Mater. 50, 207 (2004).

[14] X. Ren and K. Otsuka, Phys. Rev. Lett. 85, 1016 (2000).

[15] B. Ludwig, Ph.D. thesis, RWTH Aachen University, 2009, http://darwin.bth.rwth-aachen.de/opus3/volltexte/ 2009/2978/.

[16] T. Ohba, Y. Emura, and K. Otsuka, Mater. Trans., JIM 33, 29 (1992), http://www.jim.or.jp/journal/e/33/01/29.html.

[17] T. Ohba, S. M. Shapiro, S. Aoki, and K. Otsuka, Jpn. J. Appl. Phys. 33, L1631 (1994).

[18] T. Ohba, S. Raymond, S. M. Shapiro, and K. Otsuka, Jpn. J. Appl. Phys., Part 2, No. 1A/B, 37, L64 (1998).

[19] T. Ohba, M. Sato, and K. Otsuka, Mater. Sci. Forum 327328, 377 (2000).

[20] S. Brauer, G. B. Stephenson, M. Sutton, R. Brüning, E. M. Dufresne, S. G. J. Mochrie, G. Grübel, J. Als-Nielsen, and D. L. Abernathy, Phys. Rev. Lett. 74, 2010 (1995).

[21] A. Fluerasu, M. Sutton, and E. M. Dufresne, Phys. Rev. Lett. 94, 055501 (2005).

[22] M. Leitner, B. Sepiol, L.-M. Stadler, B. Pfau, and G. Vogl, Nature Mater. 8, 717 (2009).

[23] O. G. Shpyrko et al., Nature (London) 447, 68 (2007).

[24] L.-M. Stadler, B. Sepiol, J. W. Kantelhardt, I. Zizak, G. Grübel, and G. Vogl, Phys. Rev. B 69, 224301 (2004).

[25] M. Fromm, U. Klemradt, G. Landmesser, and J. Peisl, Mater. Sci. Eng. A 273-275, 291 (1999).

[26] S. M. Shapiro, B. X. Yang, Y. Noda, L. E. Tanner, and D. Schryvers, Phys. Rev. B 44, 9301 (1991).

[27] L. Müller, Zeitabhängige Phänomene in Athermischen Formgedächtnislegierungen (SVH, Saarbrücken, 2009), ISBN 978-3838110820. 
[28] A. Malik, A. R. Sandy, L. B. Lurio, G. B. Stephenson, S. G. J. Mochrie, I. McNulty, and M. Sutton, Phys. Rev. Lett. 81, 5832 (1998).

[29] See Supplemental Material at http://link.aps.org/ supplemental/10.1103/PhysRevLett.107.105701 for videos showing the time dependence of the scattering image.
[30] G. Brown, P. A. Rikvold, M. Sutton, and M. Grant, Phys. Rev. E 56, 6601 (1997).

[31] M. Aspelmeyer, U. Klemradt, L. T. Wood, S. C. Moss, and J. Peisl, Phys. Status Solidi A 174, R9 (1999).

[32] K. Otsuka and X. Ren, Intermetallics 7, 511 (1999). 